\title{
Compromiso estudiantil y responsabilidad social universitaria: una experiencia en la Universidad Politécnica de Pénjamo
}

\section{Student commitment and university social responsibility: an experience at the Polytechnic University of Pénjamo}

VÁZQUEZ-OLARRA, Glafira†*, ARROYO-ARROYO, Ignacio, SANTAMARÍA-RAMÍREZ, Yuridia, y RUELAS-SANTOYO, Edgar Augusto

Universidad Politécnica de Pénjamo. Licenciatura en Administración y Gestión Empresarial. Carretera Irapuato, A La Piedad Km 44, 36900 El Derramadero, Gto.

ID 1 ${ }^{\mathrm{er}}$ Autor: Glafira, Vázquez-Olarra / ORC ID: 0000-0002-1025-6785, CVU CONACYT ID: 230504

ID $1^{\text {er }}$ Coautor: Ignacio, Arroyo-Arroyo / ORC ID: 0000-0001-9128-9927

ID $2^{\text {do }}$ Coautor: Yuridia, Santamaría-Ramírez / ORC ID: 0000-0002-5468-5223, CVU CONACYT ID: 341494

ID $3^{\text {er }}$ Coautor: Edgar Augusto, Ruelas-Santoyo / ORC ID: 0000-0003-0515-7667, CVU CONACYT ID: 320367

DOI: $10.35429 / J U P .2019 .7 .3 .1 .8$

Recibido: 08 de Enero, 2019; Aceptado 28 de Marzo, 2019

\section{Resumen}

La responsabilidad social es un deber de las organizaciones, de la misma forma, las Universidades han participado en la corriente de la Responsabilidad Social, como parte de su esencia misma. Para González (2014) la Responsabilidad Social Universitaria (RSU) garantiza la calidad de la educación superior en su conjunto. En esta investigación se analiza a la Universidad Politécnica de Pénjamo (UPPE) como institución educativa, para visualizar las opciones para implementar un modelo de Responsabilidad Social Universitaria. Particularmente, se presenta el Decálogo de los Estudiantes, que constituye uno de los resultados de la investigación realizada en torno a la RSU. La investigación tiene el objetivo de determinar las opciones para implementar, dentro de la Universidad Politécnica de Pénjamo, un modelo de Responsabilidad Social Universitaria, lo que se logró a través de los resultados de la aplicación de los instrumentos propuestos por Vallaeys en el Manual de Primeros Pasos para la RSU. Los resultados contribuyen al aprendizaje a través de los estudios de caso para mostrar que las IES realizan diversas acciones desde el interior para desarrollar en los estudiantes la sensibilidad, conciencia y corresponsabilidad en la solución de los problemas del entorno.

Responsabilidad social universitaria, Estudiantes, Decálogo ético

\begin{abstract}
Social responsibility is a duty of organizations, in the same way, Universities have participated in the flow of Social Responsibility, as part of their very essence. González (2014) the University Social Responsibility (RSU) guarantees the quality of higher education as a whole. This research analyzes the Polytechnic University of Pénjamo (UPPE) as an educational institution, to visualize the options to implement a model of University Social Responsibility. In particular, the Student Decalogue is presented, which is one of the results of the research carried out around the RSU. he research aims to determine the options to implement, within the Polytechnic University of Pénjamo, a model of University Social Responsibility, which was achieved through the results of the application of the instruments proposed by Vallaeys in the First Steps Manual for the RSU. The results contribute to learning through case studies to show that IES perform various actions from within to develop in students the sensitivity, awareness and co-responsibility in solving the problems of the Environment.
\end{abstract}

University social responsibility, Students, Ethical decalogue

Citación: VÁZQUEZ-OLARRA, Glafira, ARROYO-ARROYO, Ignacio, SANTAMARÍA-RAMÍREZ, Yuridia, y RUELAS-SANTOYO, Edgar Augusto. Compromiso estudiantil y responsabilidad social universitaria: una experiencia en la Universidad Politécnica de Pénjamo. Revista de Políticas Universitarias. 2019. 3-7: 1-8.

\footnotetext{
* Correspondencia del Autor (correo electrónico: gvazquez@ uppenjamo.edu.mx)

$\dagger$ Investigador contribuyendo como primer autor.
} 


\section{Introducción}

El presente escrito forma parte de la investigación realizada en la Universidad Politécnica de Pénjamo en torno a la Responsabilidad Social Universitaria (RSU) y la comunidad estudiantil. Se realizó un estudio exploratorio-descriptivo para generar un Modelo de Responsabilidad Social Universitaria y los resultados derivaron en diferentes acciones para fortalecer la RSU. Al ser una investigación descriptiva carece de hipótesis o supuestos. Se muestra que la investigación-acción sigue siendo fundamental en la transformación de los entornos para generar cambios específicos, solucionen un problema o bien, satisfagan una necesidad en el corto plazo.

Se presenta un segmento de la investigación describiendo la necesidad de la Universidad; el diseño de investigación y los resultados orientados hacia el compromiso estudiantil a través del Decálogo de Responsabilidad Social de los estudiantes y los ecodiseños realizados por los mismos alumnos. Finalmente, se muestran las conclusiones y los desafíos que representa la inserción de la RSU en el contexto y hábitos de los estudiantes.

\section{Descripción del problema}

En los últimos tiempos las empresas, en general, han obtenido un gran interés por implementar la responsabilidad social dentro de sus actividades diarias ya que estas prácticas forman parte de su estrategia corporativa ya que tienen como fin evitar daños y/o producir beneficios para sus partes interesadas (clientes, empleados, accionistas, comunidad, entorno, etc.), siguiendo fines que deben tener un beneficio tanto para la organización como para la sociedad. De igual manera, las Universidades se han interesado en el tema de Responsabilidad Social, han reaccionado ante la necesidad transformar paulatinamente sus estructuras de gestión, formación e investigación para adaptarlas a las exigencias de este contexto competitivo.

La misma pregunta por la responsabilidad de las instituciones de la Educación Superior en nuestra sociedad es una pregunta que confirma, hasta cierto punto, que la universidad es consciente de ese proceso de cambio.
Y las respuestas que está dando son manifestación de su interés por formar parte activa de ese proceso y de los ámbitos donde se están decidiendo las líneas básicas de actuación de la Educación Superior en las próximas décadas (Vallaeys, 2013).

La Responsabilidad Social Universitaria (RSU) surge como alternativa para emprender en una etapa en donde se tenga como base un nuevo paradigma de aprendizaje y gestión del conocimiento a lo largo de toda la vida, y asumiendo la responsabilidad social como premisa para construir sociedades inclusivas del conocimiento, y un desarrollo sustentable para todos los países de la región (Aponte, 2008).

Gestionar la Universidad con criterios socialmente responsables no debe responder a las leyes de la economía y del mercado, asumiendo al estudiante como un cliente, y a la educación superior como un producto, porque esa concepción es inapropiada -por decir lo menos- en el quehacer universitario. El modelo de desarrollo actual es insostenible a nivel económico, ambiental y social, y la universidad debe contribuir a cambiar esa realidad.

La universidad como organización legendariamente ligada al desarrollo de la sociedad, debería asumir un modelo de gestión basado en responsabilidad social. La Responsabilidad Social Universitaria -RSUdinamizará el funcionamiento interno de la organización, y facilitará el diálogo y la construcción de vínculos con otros actores sociales, para juntos articularse a un proyecto planificado de desarrollo sostenible de la sociedad. La delicada misión de la universidad como agente de desarrollo social, toma mayor relevancia en la denominada sociedad del conocimiento que vivimos. En esta nueva estructura económica y social, el conocimiento sustituye al trabajo, a las materias primas y al capital, e impacta a la sociedad. El principio de que la producción académica y científica que se gesta en las universidades debe transferirse a la sociedad, se garantiza con la adopción de un modelo de gestión socialmente responsable, que por esencia, motiva la participación e interlocución con el entorno (González, 2014). 
Particularmente, esta investigación se realiza en la Universidad Politécnica de Pénjamo (UPPE) como institución educativa; a la comunidad académica y estudiantil de la Licenciatura en Administración y Gestión Empresarial, siendo clara la intención de la UPPE por llegar a ser una Universidad Socialmente Responsable. En la Misión de la UPPE se menciona: Somos una institución educativa, formadora de personas íntegras, que consolidan un proyecto de vida, con sentido y compromiso social, capaces de influir en el crecimiento competitivo de la región.

Es posible decir que el estudiante aprende su carrera en la Universidad, pero, también, aprende de la Universidad los hábitos y valores ciudadanos que se deben poner en práctica, ya que cada acción realizada internamente tiene un gran impacto en la sociedad en general.

Se puede clasificar a la Universidad como aquel filtro por donde pasa la mayoría de los empresarios y funcionarios públicos, lo cual aumenta la responsabilidad de la Universidad para formar a los verdaderos profesionistas y personas que la sociedad necesita hoy en día. (Martí-Noruega, 2018).

Debido al crecimiento de la Universidad y el aumento de la matrícula de alumnos, se ha observado que los espacios comunes para la actividad estudiantil son insuficientes, de ello deriva, las acciones mostradas en este escrito.

Como resultado de la necesidad de fortalecer la formación y hábitos de los estudiantes y su compromiso social, se requiere definir acciones que fortalezcan la identidad universitaria en los estudiantes de la UPPE; por lo tanto, el objetivo de la investigación es: determinar las acciones que promuevan el involucramiento de los estudiantes la UPPE en el marco de la RSU y alineadas a la filosofía universitaria. Enseguida se muestra un breve contexto de la Responsabilidad Social Universitaria.

\section{Marco Teórico}

\section{¿Qué es la responsabilidad social universitaria?}

Las universidades se constituyen en una red, compuesta por todos los miembros de la comunidad universitaria.
Se organizan además en torno a una misión que queda concretada en un conjunto de normas de convivencia y en un proyecto universitario que define su identidad y sus valores. Se espera que todos los miembros de la comunidad universitaria asuman, compartan y promuevan esos valores. Y se espera también que la sociedad reconozca esa identidad y esos valores como propios de la universidad. Es decir, se espera que la sociedad legitime lo que la universidad quiere ser y la manera en la que quiere estar y ser sociedad. (Vallaeys, 2013)

La universidad participa, en la esfera pública, en la construcción de ciudadanía, contribuye al desarrollo de políticas públicas concretas participando mediante lo que le es más propio: formación y la generación y transmisión de nuevos conocimientos. En esa esfera pública, la universidad es (o debiera ser) un observatorio crítico de la realidad social y en ella es reconocida como un interlocutor necesario (un stakeholder) para el diálogo social, reconozcamos, por tanto, su valor transformador y hagamos pivotar su responsabilidad precisamente en él como el más propio para el desarrollo de su Misión. (Vallaeys, 2013)

La RSU se constituye en un compromiso activo, integral, real con el entorno interno y externo de la universidad, tomando en cuenta la trascendencia de la universidad como motor de la sociedad; las características propias que el cumplimiento de las dimensiones universitarias conlleva en la sociedad del conocimiento; $y$, sus impactos particularmente importantes $y$ diferentes, a las de cualquier otra organización de la sociedad.

Por lo tanto, es posible decir que la universidad tiene funciones distintas a la empresa, por lo tanto, sus compromisos son diferentes.

La universidad busca formar ciudadanos profesionales e investigadores que actúen sobre la diversidad de problemas de la sociedad, y además sobre la transformación de la misma, lo cual difiere de los objetivos de otras entidades. (González, 2014) 


\section{La Responsabilidad Social Universitaria como nuevo contrato social para la Universidad}

Para dibujar la visión a largo plazo que se quiere instituir con la Responsabilidad Social Universitaria, queremos indicar algunas ideas que podrían servir de modelo para diseñar un nuevo "contrato social" entre la Universidad y la sociedad. Los diversos puntos que planteamos para este "nuevo contrato social" sólo pretenden estimular la propia reflexión y nutrir el debate entre académicos, responsables universitarios, estudiantes y la sociedad civil. Conviene que cada Universidad y cada grupo docente diseñen su propia política de acción en relación con su propia identidad y contexto social, (Vallaeys, 2009), los puntos son:

- Garantizar la Responsabilidad Social de las Ciencias.

- Promover la formación a la ciudadanía democrática.

- Educar al estudiante como un agente de desarrollo.

Se puede formular el nuevo contrato social entre la Universidad y la Sociedad en estos términos:

"Tú, Sociedad, me garantizas autonomía y recursos, y yo, Universidad, te doy (1) Más Democracia a través de la formación de estudiantes y ciudadanos responsables, (2) más Ciencia responsable, lúcida y abierta a la solución de los problemas sociales de la humanidad, y (3) mejor Desarrollo equitativo, innovador y sostenible, con profesionales competentes y comprometidos." (Vallaeys, s.f.).

Otro autor que aborda a la Responsabilidad Social en la Universidad es Víctor Corral, quien menciona que la Universidad Sustentable (US) cuida el ambiente socio-físico; esto lo logra a través de prácticas de conservación de su campus: las que incluyen: cuidado del agua, energía, suelo, aire, recursos naturales. Para la parte de la conservación del entorno social, la US estimula una cultura para la paz (procesos de mediación, respeto a derechos humanos, fomento a la cooperación y la conducta pro social) (Corral, 2016).
Una Universidad Sustentable (US) satisface las necesidades de sus integrantes; ayuda a satisfacer las necesidades de la comunidad a la que sirve; conserva el ambiente socio físico inmediato y colabora a cuidar su capital humano, natural, social, manufacturado y financiero. (Corral, 2016)

La responsabilidad social no admite parcialidad ni segmentaciones: no se pueden desarrollar actuaciones responsables en un ámbito y dejar otros ocultos a la mirada ética. Por lo tanto, no se trata sólo de crear una nueva oficina de RSU o de mejorar el área de extensión y la proyección social solidaria de la universidad sin tocar a los demás procesos de la institución. La responsabilidad social debe lograr colorear y capilarizar a toda la universidad. Uno de sus valores más importantes es precisamente la coherencia institucional, que significa a la vez coincidencia entre la acción y el discurso institucional y consistencia entre todas las áreas de la universidad (que no haya contradicción entre lo que hace un área y lo que hace otra).

Por lo tanto, el proceso de responsabilidad social alcanza los cuatro ámbitos de la universidad:

1. Ámbito organizacional: en tanto institución que opera en torno a un proyecto universitario, con una estructura que lo desarrolla y unas políticas concretas que lo promueven. Una institución, además, que consume, contrata, genera residuos, etcétera.

2. Ámbito educativo: en tanto institución que se encarga de la formación de sus estudiantes, con una vocación profesionalizante y cívica.

3. Ámbito del conocimiento: en tanto institución que investiga, que produce saber y lo transmite.

4. Ámbito social: en tanto institución que forma parte de la sociedad e interactúa con otros agentes, colectivos y comunidades, tanto a nivel local como global.

\section{Los beneficios de la responsabilidad social para la universidad}

La responsabilidad social universitaria conlleva varios beneficios a corto y largo plazo. 
- Coherencia e integración institucional: la RSU ayuda a la universidad a articular sus funciones sustantivas de docencia, investigación y extensión mediante una política transversal que capitaliza toda la institución y alinea los diversos procesos académicos y no académicos con un enfoque de gestión ética responsable. Permite lograr una coherencia entre las declaraciones de intenciones (misión, visión y valores institucionales) y la práctica cotidiana en el campus.

- Pertinencia y permeabilidad social: la RSU ayuda a la universidad a abrirse a su entorno social; convoca a actores externos para participar en los procesos académicos y organizacionales internos, y orienta la gestión, la formación y la investigación hacia la solución de problemas sociales concretos. Asimismo, es una política institucional adecuada para el tratamiento de la problemática global de insostenibilidad social y ambiental que nos toca enfrentar en el presente siglo.

- Dinámica institucional hacia la innovación: la RSU ayuda a la universidad a ser una organización inteligente, que se piensa a sí misma en forma transparente y democrática, y que implementa en su seno procesos de mejora continua que facilitan iniciativas creativas en los ámbitos académicos y de gestión.

- Racionalización de la gestión universitaria: la RSU ayuda a la universidad a mejorar el desempeño de sus diversos procesos, desde la gestión racional del campus (manejo ambiental) hasta el incremento de la motivación (empoderamiento de las personas), pasando por el mejoramiento del rendimiento académico (pertinencia social de la enseñanza e investigación) y la creación de valor social (proyectos de desarrollo en la comunidad). (Vallaeys, 2009)

Con base en estos referentes se aplicó la metodología siguiente.

\section{Metodología}

\section{Diseño de investigación}

El diseño de la presente investigación es no experimental, transversal, exploratorio y descriptivo, debido a que no se manipuló ninguna variable, solamente se relatan las condiciones en que se encuentra la Universidad en torno a la RSU. Se recopilaron los datos durante el último cuatrimestre de 2018.

Asimismo, se aplicó el diseño llamado: investigación-acción, mismo que, de acuerdo con Hernández (2010) afirma que la finalidad de la investigación-acción es resolver problemas cotidianos e inmediatos (Álvarez-Gayou, 2003; Merriam, 2009) y mejorar prácticas concretas. Es decir, se investiga y se interviene para generar cambios en el entorno, esto se realizó a través de las acciones para crear ecodiseños y manejo de la basura en las aulas, para cambiar hábitos y migrar hacia el enfoque sustentable.

\section{Recolección de datos}

La recopilación de los datos se realizó a través de 3 encuestas dirigidas a los distintos actores de la universidad (alumnos, personal docente, personal no docente) con la finalidad de cubrir los 4 ejes de la Responsabilidad Sociales Universitaria que se manejan según Vallaeys.

Los cuatro ejes de responsabilidad que corresponden a las cuatro áreas de impacto: Campus responsable, Formación profesional y ciudadana, Gestión social del conocimiento, Participación social.

\section{Resultados}

Con base los resultados de los cuestionarios aplicados a los estudiantes, se detectó la necesidad de generar un Modelo de Responsabilidad Social Universitaria basado en los cuatro pilares fundamentales y generales que presenta Vallaeys en su concepto de RSU. En paralelo, se hizo una propuesta para establecer el Decálogo de Responsabilidad Social de los y las estudiantes de la Universidad Politécnica de Pénjamo, el cual dirige las acciones y genera el compromiso del estudiantado de la UPPE, desde un enfoque cívico y ético. 
Asimismo, el Decálogo de Responsabilidad Social pretende crear conciencia entre el alumnado para orientar su comportamiento hacia acciones concretas sociales y sustentables.

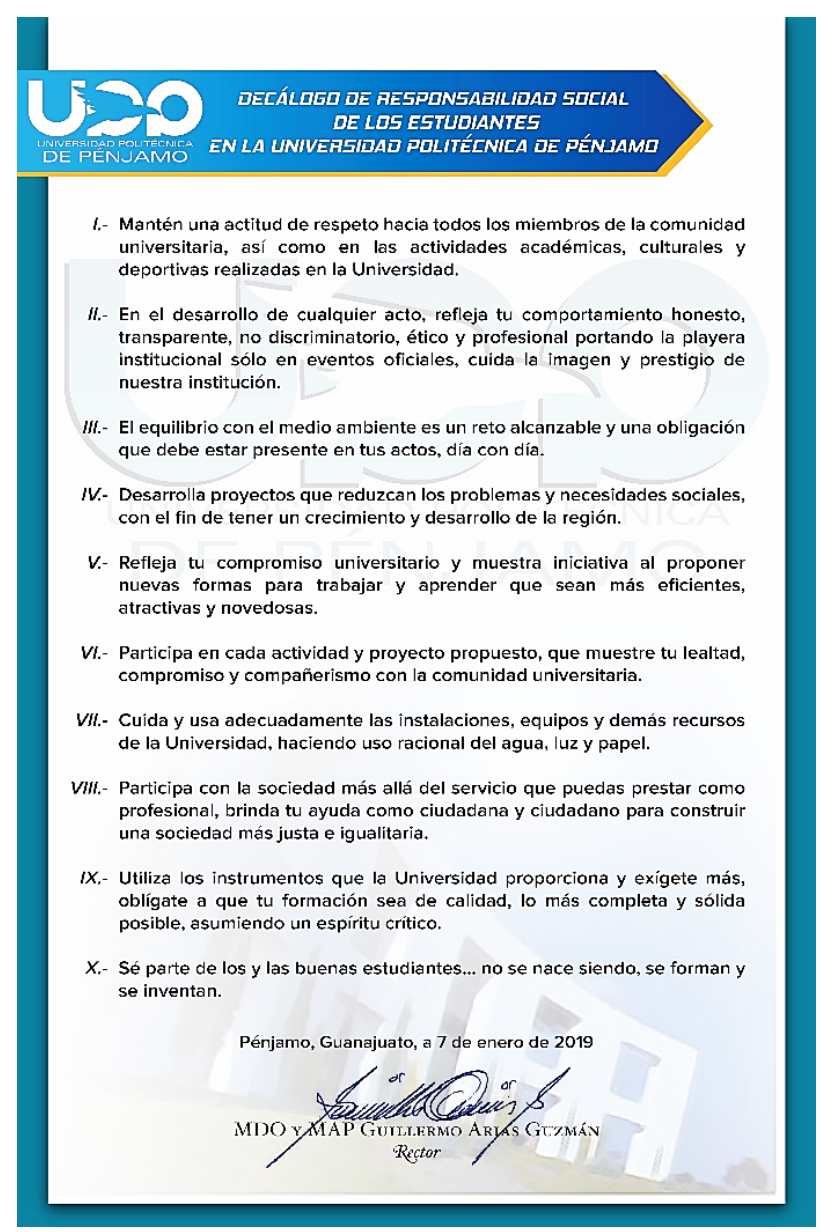

Figura 1

Durante el proceso de la investigaciónacción se observó que los estudiantes se interesaron en unirse a las acciones sustentables y realizaron invitaciones a amigos y compañeros para elaborar muebles con material de reuso, lo cual permitió que, en cuatro meses, se elaboraran un promedio de 15 bancas para ser usadas por los estudiantes.

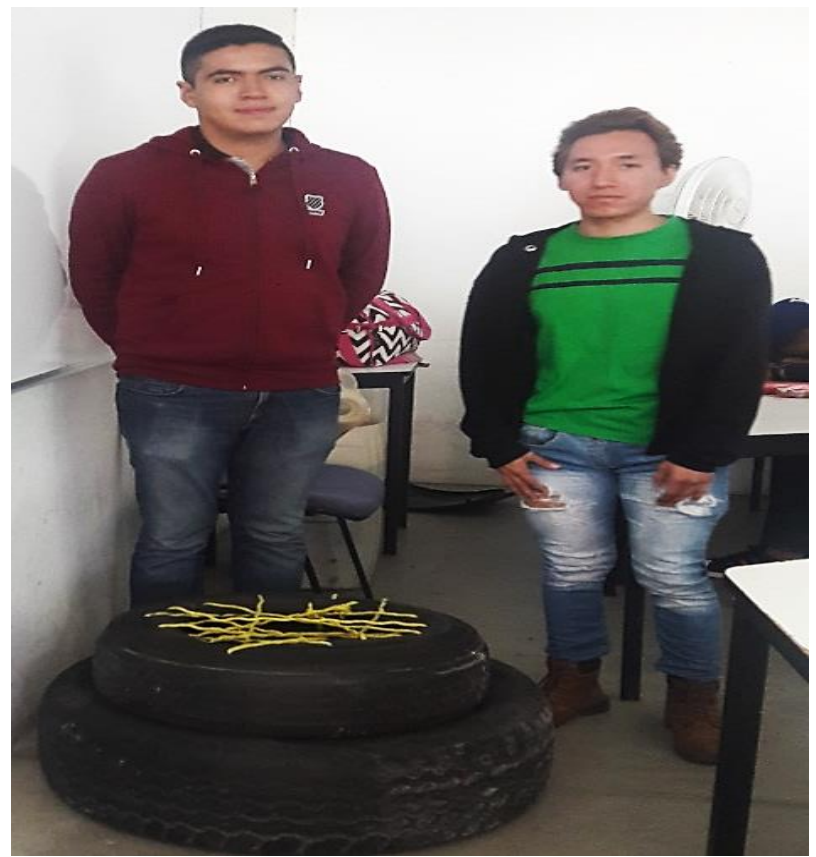

Figura 2

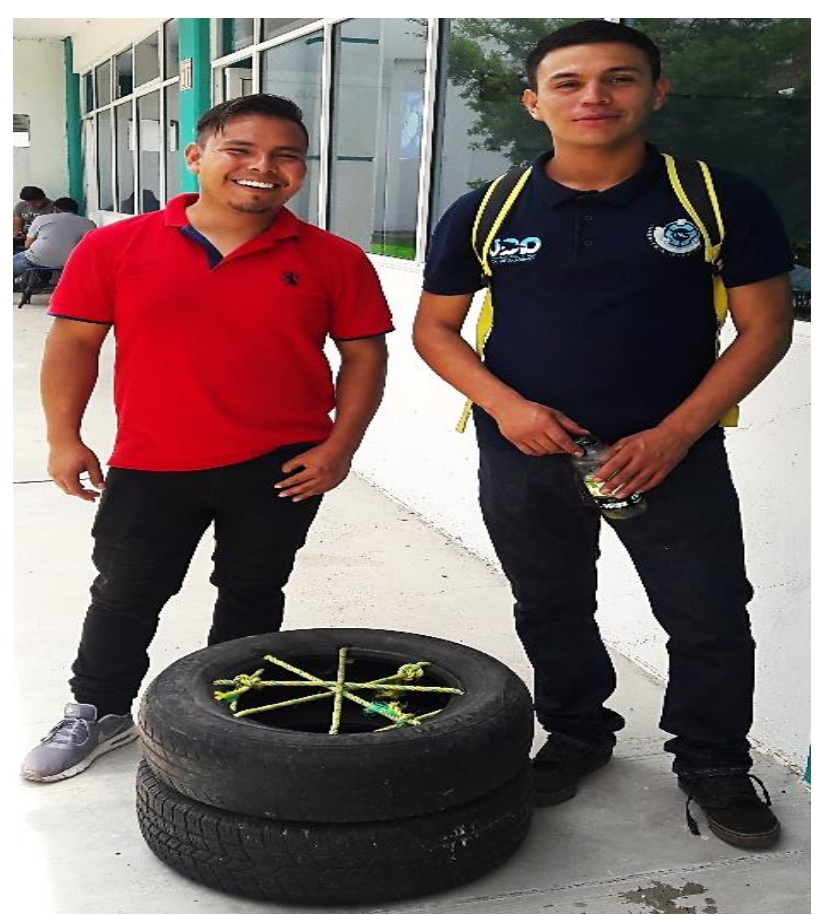

Figura 3

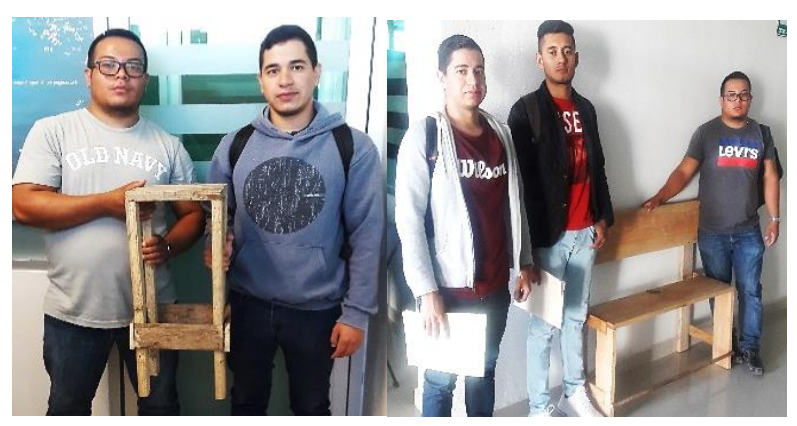

Figura 4 


\section{Agradecimiento}

Externamos nuestro agradecimiento a la Universidad Politécnica de Pénjamo por apoyar las iniciativas de maestros y estudiantes.

\section{Conclusiones}

La Universidad Politécnica de Pénjamo al ser parte de las universidades politécnicas instituciones públicas comprometidas a contribuir en el desarrollo social y económico del país cuenta con lineamientos claros que le ayudan a contar con una filosofía responsable, ética, sustentable y por supuesto, siempre se puede mejorar.

La UPPE en sus pocos años de formación ha tenido un crecimiento significante, sin embargo, se han descuidado algunos puntos importantes, pues además de ofrecer una educación de calidad se debe ofrecer un servicio de calidad al personal, proveedores y gobierno, sobre todo impactar de forma positiva la sociedad que le rodea, cuidar que los proyectos que realizan sus alumnos contribuyan al medio ambiente.

Recordar que se enfocan en tres saberes $\mathrm{y}$, por ende, debe 'entregar' a la sociedad profesionistas con una ética incomparable, hacerse reconocer como una universidad humanista, una universidad socialmente responsable. El que la UPPE cuente con una filosofía total de RSU traería beneficios como: reconocimiento social y preferencia a su educación aumentando su matrícula con razón justificada y logrando que cada una de los estudiantes ingresados egresen satisfactoriamente, docentes y demás personal más convencidos, motivados y por lo tanto más capacitados y dispuestos a entregar un trabajo de calidad y excelencia humana.

Se espera que, en la medida en que el estudiante vive el Decálogo de Responsabilidad Social desarrolle el sentido de Identidad Universitaria, tome su rol activo de estudiante universitario, gestor de cambios entre sus pares académicos y en la carrera profesional que cursa, de tal forma que esas acciones se conviertan en un estilo de vida universitario y, el impacto de su profesión sea en y fuera del aula para beneficiar su entorno.
Durante el periodo de realización de esta investigación se generaron acciones orientadas hacia el cuidado ambiental y concienciación de las acciones sustentables creando ecodiseños; en atención a la Racionalización de la gestión universitaria, como señala Vallaeys (2013) se logró el interés y empoderamiento de los estudiantes, así como la creación de valor social, al desarrollar acciones ambientales y de creación de ecomuebles o ecodiseños, elaborados con material de reuso, donde los mismos estudiantes suministraron el material y fabricaron los muebles.

Con ello, la universidad aporta personas comprometidas con las necesidades del entorno desde el enfoque de la sustentabilidad, el aprovechamiento de los recursos y la satisfacción de necesidades, siendo los estudiantes, agentes de desarrollo.

Se comprueba que la Universidad Sustentable, tal como la menciona Corral, cuida el ambiente socio físico a través de diversas prácticas, en este caso, al cuidado de los recursos naturales; asimismo, se fomentó la conducta Prosocial a través de la solidaridad y empatía guiando al estudiante a generar soluciones ante la falta de espacios comunes para consumo de alimentos, recreación y discusión académica.

El inicio de estas acciones es una muestra de la Responsabilidad Social Universitaria desarrollada de la Universidad Politécnica de Pénjamo, cumpliendo con su lema:

"... compromiso social para trascender."

\section{Referencias}

Corral, V. (2016). Sustentabilidad, psicología y responsablidad social universitaria. En E. Rivera, R. Vallejo, A. Méndez, González, \& F. (Coord), Jóvenes, psicología y responsabilidad social universitaria. (págs. 43-58). México: CUM, UMSNH, Porrúa.

González. (2014). Responsabilidad Social Universitaria. Apuntes para un modelo de RSU. Revista de Comunicación 13, 84-117.

Hernández, R. F., \& Baptista, P. (2014). Metodología de la investigación (6a ed.). México, D.F., México: Mc Graw Hill. 
Martí-Noruega, J. (10 de Septiembre de 2018). ¿Cómo se ha abordado la Responsabilidad Social en Instituciones de Educación Superior en México y América Latina? Jornadas de Responsabilidad Social 2018. San Miguel de Allende, Guanajuato, México.

Vallaeys, F. (2009). Responsabilidad Social Universitaria: Manual de primeros pasos. México, D.F.: Mc Graw -Hill Interamericana.

Vallaeys, F. (2013). El movimiento de responsabilidad social de la universidad: Una compresión novedosa de la misión universitaria. Educación Superior y Sociedad, 191-219.

Vallaeys, F. (s.f.). ¿Qué es la Responsabilidad Social Universitaria? (P. U. Perú, Ed.) Recuperado el 11 de septiembre de 2018, de http://creasfile.uahurtado.cl/RSU.pdf 